\title{
Electromechanical Modeling and High Speed Design of a Tubular Ultrasonic Motor
}

\author{
Vahid Dabbagh \\ Centre of Advanced Manufacturing and Material Processing, Department of Mechanical Engineering, Faculty of \\ Engineering, University of Malaya, 50603, Kuala Lumpur, Malaysia.
}

\author{
Ahmed A. D. Sarhan \\ Centre of Advanced Manufacturing and Material Processing, Department of Mechanical Engineering, Faculty of \\ Engineering, University of Malaya, 50603, Kuala Lumpur, Malaysia. \\ Department of Mechanical Engineering, Faculty of Engineering, Assiut University, Assiut 71516, Egypt.
}

Javad Akbari

School of Mechanical Engineering, Sharif University of Technology, Tehran, 14588-89694, Iran.

\begin{abstract}
N. A. Mardi
Centre of Advanced Manufacturing and Material Processing, Department of Mechanical Engineering, Faculty of Engineering, University of Malaya, 50603, Kuala Lumpur, Malaysia.
\end{abstract}

\section{(Received 8 January 2016; accepted 5 October 2016)}

Tubular ultrasonic motors are one of the simplest yet effective methods to realize rotational ultrasonic motors. In this paper, electromechanical modeling of a tubular ultrasonic motor is achieved using assumed-modes method, Euler-Bernoulli beam theory, and constitutive equations of piezoelectricity. Upon validating the analytical model using finite element method (FEM) and the experimental result, effect of the design variables on speed is investigated using sensitivity analysis. The result proves that the speed of the motor is almost independent of the tube length for a constant length ratio of the tube and piezo-plates. Excellent agreement between the experimental and analytical model is observed. In addition, a set of guidelines for high speed designing of the stator is proposed. The results of this paper greatly facilitate design of tubular ultrasonic motors with respect to achieving high speed motor.

\section{INTRODUCTION}

Ultrasonic motors, transducers that convert ultrasonic vibrations to the rotational or linear movement, are valuable for the industry and researchers due to their superior characteristics compared to the conventional electromagnetic motors. Some of the advantages are the ability of miniaturization, simple structure, high reliability, and functioning capability in high magnetic field and vacuum spaces such as in a spacecraft. ${ }^{1}$

Tubular ultrasonic motors (TUSM) are designed to produce rotary movement in a simple and straightforward way. Two types of travelling and standing wave tubular ultrasonic motors are categorized and investigated by researchers. ${ }^{2-9}$ Attached piezoelectric transducers to the stator convert alternative voltage signal to vibration. Thereby, the resulted tooth displacements by stator vibrations rotates the shaft through friction force.

Parametric optimization of ultrasonic motors intended to enhance the characteristics of motors such as speed, torque, and efficiency is one of the main fields of research in ultrasonic motor designs. Pons et al. ${ }^{10}$ proposed a new optimization approach for the stator of travelling ultrasonic motors, according to the stator, rotor, and interference model. Zhao et al. ${ }^{11}$ in- troduced an optimization design method for a three-degree of freedom ultrasonic motor using constrained variable metric algorithm (CVMA). Mathematical models combined with finite element method (FEM) is used for estimating the characteristics of a motor. Experimental mode shapes and frequencies were in good agreement with those predicted by the program. Ko et al. ${ }^{12}$ investigated the curvature of the stator tip and its normal force along with the stress analysis to improve torque, speed, transmitted power, and efficiency. Eventually they validated the influence of the stator's tip on the motor characteristics using experimental data. Zhu et al. ${ }^{13}$ prepared the electromechanical coupled dynamic model of bar-type ultrasonic motor based on finite element method. An optimal model to achieve design requirement was derived using the pattern search algorithm. Zhang et al. ${ }^{14}$ accomplished multi-objective optimization of rod shape ultrasonic motor using sequential quadratic programming and finite element method. The proposed design method achieved significant enhancement in the design indexes and was effective in the design of ultrasonic motors.

This paper concerns the electromechanical modeling, sensitivity analysis, and the design of tubular ultrasonic motors, which aim to increase motor speed. Main dimensions of the 


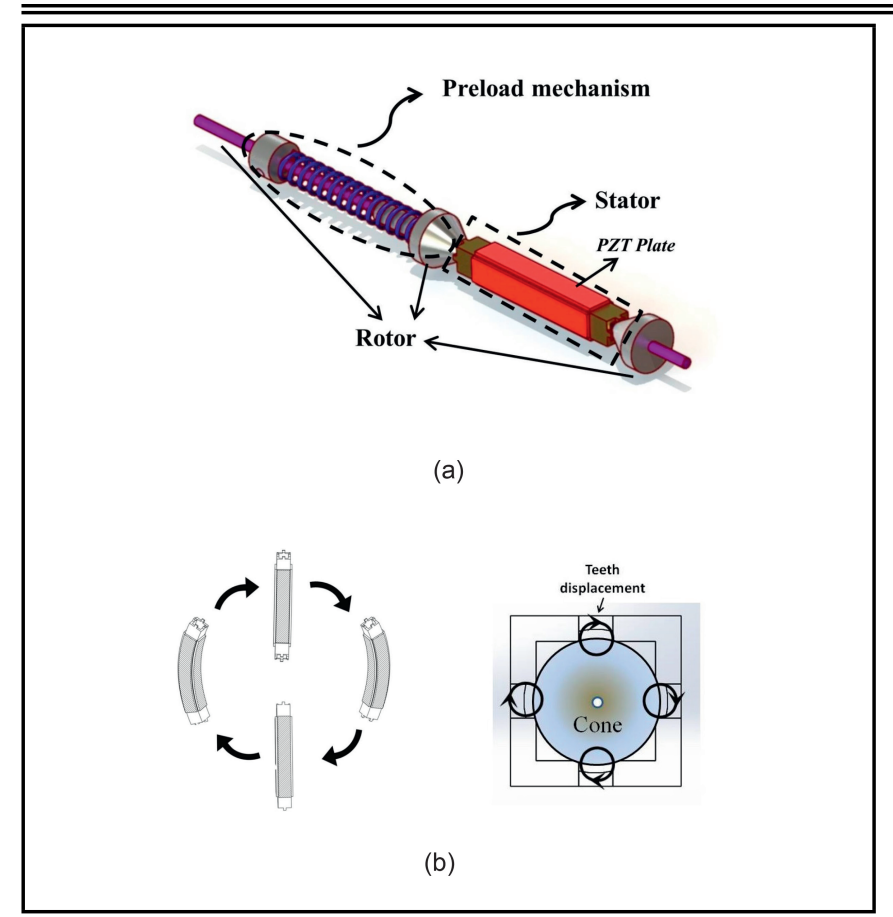

Figure 1: Tubular ultrasonic motor (a) stator, rotor and preload of TUSM, (b) Illustration of the procedure that motor converts bending ultrasonic vibration to rotational motion of the rotor.

tube and piezo-plates are considered as design variables and their effects on the speed of the motor are investigated using analytical model and sensitivity analysis. Finally, a guideline for design of the motor to achieve high speed is proposed and the main points are explained.

\section{ELECTROMECHANICAL MODELING OF THE STATOR OF A TUBULAR ULTRASONIC MOTOR}

\subsection{Tubular Ultrasonic Motor}

A tubular ultrasonic motor is composed of a stator, rotor, and preload mechanism as shown in Fig. 1a. In this figure, the stator is composed of piezoelectric ceramic plates that are connected using an adhesive layer to a metallic tube. As a result of applying a sinusoidal voltage on the piezoelectric plates with a frequency near the first bending mode, the stator will convert electric voltage to mechanical vibration shown in Fig. 1 b. Energy from this vibration is transferred through the contact of the tooth between the stator and the rotor, and eventually causes rotation of the rotor. Further description of this motor is discussed by Park and He. ${ }^{8}$ In the following section we develop an approximated analytical solution using assumedmodes formulation, Euler-Bernoulli beam theory, and constitutive equations of piezoelectricity.

Figure 2a shows the electric circuit used for the excitation of the stator. A sinusoidal voltage $v_{S}$ is generated with a voltage source with an internal resistance of $R$. In Fig. 2b, the length of the tube and piezo-plates, the width of tube and piezo-plates, and the thickness of the tube are denoted by $L_{t b}, L_{p}, a_{t b}, a_{p}$, and $t$, respectively. The system of coordinates is located on the left side of the stator where $x_{1}$ coincides with the neutral

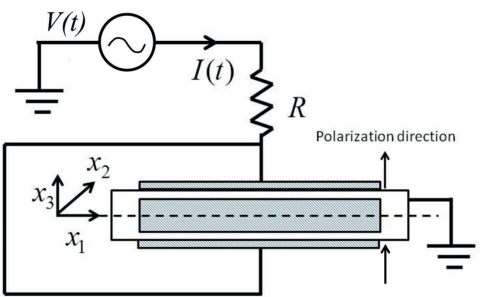

(a)

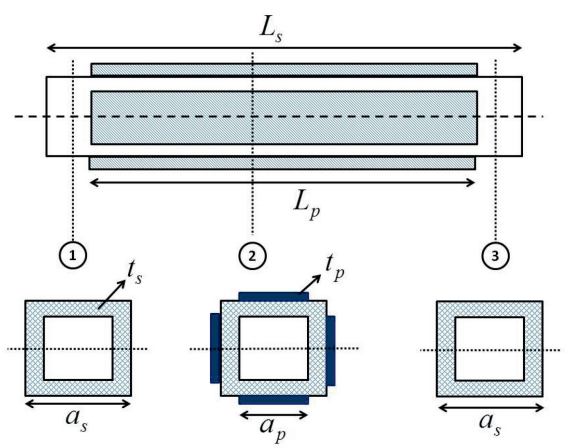

(b)

Figure 2: Stator of TUSM, (a) electric circuit for excitation of the motor (b) Main geometrical parameters of the stator and its cross section.

axis of the stator. Directions of polarization of the piezo-plates oppose each other.

\subsection{Extended Hamiltonian's Principle and Lagrange's Equations}

Because of the difficulty associated with the section variation along the stator, Hamiltonian's principle is utilized to derive an approximated solution for electromechanical vibration of the stator. Extended Hamiltonian's principle considering electrical energy stored in the piezo-plates is: ${ }^{15}$

$$
\int_{t_{1}}^{t_{2}}\left(\delta T-\delta U+\delta W_{i e}+\delta W_{n c}\right) d t=0 ;
$$

where $\delta T, \delta U, \delta W_{i e}$, and $\delta W_{n c}$ are first variation of total kinematic energy, total elastic potential energy, internal electric energy stored in the piezo-plates, and energy dissipation due to the structural damping of the stator, respectively. These terms can be addressed using generalized coordinates $q_{n}(t)$ and their time derivatives $\dot{q}_{n}(t)$ as:

$$
\begin{gathered}
T=T\left(q_{1}, q_{2}, \ldots, q_{n}, \dot{q}_{1}, \dot{q}_{2}, \ldots, \dot{q}_{n}\right) ; \\
U=U\left(q_{1}, q_{2}, \ldots, q_{n}\right) ; \\
W_{i e}=W_{i e}\left(q_{1}, q_{2}, \ldots, q_{n}\right) .
\end{gathered}
$$

Taking first variation of the energy terms in (2) and substituting in (1), integrating by parts, gives Lagrange's Eq. (3b): 


$$
\begin{gathered}
\int_{t_{1}}^{t_{2}}\left\{\sum _ { k = 1 } ^ { n } \left[\frac{\partial T}{\partial q_{k}}-\frac{\partial U}{\partial q_{k}}+\frac{\partial W_{i e}}{\partial q_{k}}-\right.\right. \\
\left.\left.\frac{d}{d t}\left(\frac{\partial T}{\partial \dot{q}_{k}}\right)+Q_{k}\right] \delta q_{k}\right\} d t=0 ; \\
\frac{d}{d t}\left(\frac{\partial T}{\partial \dot{q}_{k}}\right)-\frac{\partial T}{\partial q_{k}}+\frac{\partial U}{\partial q_{k}}-\frac{\partial W_{i e}}{\partial q_{k}}=Q_{k} ;
\end{gathered}
$$

where $Q_{k}$ is generalized non-conserved force derived by $\delta W_{n c}=\sum_{k=1}^{n} Q_{k} \delta q_{k}$.

\subsection{Lagrange's Equations for Euler-Bernoulli Beam and Piezo-Plates}

Total kinematic energy of the stator is comprised of the tube and piezo-plates kinetic energy given by:

$$
T=\frac{1}{2}\left(\int_{V_{t b}} \rho_{t b} \frac{\partial \mathbf{u}^{t}}{\partial t} \frac{\partial \mathbf{u}}{\partial t} d V_{t b}+\int_{V_{p}} \rho_{p} \frac{\partial \mathbf{u}^{t}}{\partial t} \frac{\partial \mathbf{u}}{\partial t} d V_{p}\right)
$$

where $\rho_{t b}, \rho_{p}, V_{t b}$, and $V_{p}$ are density and volume of tube and piezo-plates, respectively and superscript $t$ stands for transpose. Vector of displacement is denoted by $\mathbf{u}=$ $\left[\begin{array}{lll}u_{1} & u_{2} & u_{3}\end{array}\right]^{t}$. Assuming that the beam is vibrating in its first bending mode we can assume that the transverse and axial displacement is zero which means $\mathbf{u}=\left[\begin{array}{lll}u_{1} & 0 & 0\end{array}\right]^{t}$.

Elastic potential energy stored in the tube and piezo-plates is:

$$
U=\frac{1}{2}\left(\int_{V_{t b}} \mathbf{S}^{t} \mathbf{T} d V_{t b}+\int_{V_{p}} \mathbf{S}^{t} \mathbf{T} d V_{p}\right) ;
$$

where $\mathbf{S}$ and $\mathbf{T}$ are the strain and mechanical stress vector, respectively.

According to Euler-Bernoulli beam theory, strain vector can be related to curvature of beam by: ${ }^{16}$

$$
\mathbf{S}=\left[-x_{3} \frac{\partial^{2} u_{1}\left(x_{1}, t\right)}{\partial x_{1}{ }^{2}} \quad 0 \quad 0\right]
$$

Neglecting axial and in-plane deformation, strain vector will be: $\mathbf{S}=\left[\begin{array}{lll}S_{1} & 0 & 0\end{array}\right]^{t}$

Using Hooke's law we can relate stress to strain on the tube using the following relations:

$$
T_{1}=Y_{t b} S_{1}, \quad T_{2}=0, \quad T_{3}=0 ;
$$

where $Y_{t b}$ is the elastic modules of the tube material.

Similarly, we could obtain stress of the piezo-plates using the equation of converse piezoelectric effect: ${ }^{1}$

$$
T_{1}=Y_{p} S_{1}-e_{31} E_{3}=Y_{p}\left(-x_{3} \frac{\partial^{2} u_{1}}{\partial x_{3}^{2}}\right)+e_{31} \frac{v_{p}}{t_{p}} ;
$$

where $E_{3}$ is the electric field across thickness of the piezoplates $t_{p}$ by the external voltage of $v_{p}$. In addition, piezoelectric stress constant is denoted by $e_{31}$.

Electric energy stored in the piezo-plates depends on the vector of the electric field, $\mathbf{E}$ and electric displacement field $\mathbf{D}=\left[\begin{array}{lll}0 & 0 & D_{3}\end{array}\right]^{t}$ as follows:

$$
W_{i e}=\frac{1}{2} \int_{V_{p}} \mathbf{E}^{t} \mathbf{D} d V_{p}
$$

We can obtain electric displacement using the equation of direct piezoelectric effect: ${ }^{1}$

$$
D_{3}=e_{31} S_{1}+\epsilon_{33} E_{3}=e_{31}\left(-x_{3} \frac{\partial^{2} u_{1}}{\partial x_{1}^{2}}\right)-\epsilon_{33}^{s} \frac{v_{p}}{t_{p}}
$$

where $\epsilon_{33}^{S}$ is permittivity of piezoelectric material at constant strain.

Using assumed-modes method, the following series defines the stator transverse deformation $u_{1}(x, t)$ at a given point along the stator:

$$
u\left(x_{1}, t\right)=\sum_{r=1}^{N} a_{r}(t) \phi_{r}\left(x_{1}\right)=\{q\}^{t}\left\{\phi\left(x_{1}\right)\right\} ;
$$

where $\{a\}$ and $\left\{\phi\left(x_{1}\right)\right\}$ are the vector of generalized coordinates and shape functions that satisfy global boundary conditions, respectively, and $N$ is the total number of modes utilized. Since the stator is in free-free condition, we can use the mode shapes of free-free Euler-Bernoulli beam defined as: ${ }^{16}$

$$
\begin{aligned}
& \phi_{n}\left(x_{1}\right)=\left[\sinh \left(k_{n} x_{1} / L_{t}\right)+\sin \left(k_{n} x_{1} / L_{t}\right)\right]+ \\
& \frac{\sin \left(k_{n}\right)-\sinh \left(k_{n}\right)}{\cosh \left(k_{n}\right)-\cos \left(k_{n}\right)}\left[\cosh \left(k_{n} x_{1} / L_{t}\right)+\cos \left(k_{n} x_{1} / L_{t}\right)\right]
\end{aligned}
$$

where $k_{n}$ is solution of $\cos \left(k_{n}\right) \cosh \left(k_{n}\right)=1$.

Employing equations (4)- (11) in (3b), matricial form of Lagrange's equation for a tubular ultrasonic motor could be derived as follows:

$$
\mathbf{m}\{\ddot{a}\}+\mathbf{d}\{\dot{a}\}+\mathbf{k}\{\dot{a}\}-\{\theta\} v_{p}=0 .
$$

In the above equation, $\mathbf{m}$ and $\mathbf{k}$ are the mass and stiffness matrix and $\theta$ is the electromechanical coupling vector. The structural damping matrix, denoted by $\mathbf{d}$, could be assumed as a linear combination of the mass and stiffness matrix with proportionality constants $\mu$ and $\gamma$ as follows: ${ }^{16}$

$$
\mathbf{d}=\mu \mathbf{m}+\gamma \mathbf{k}
$$

In Eq. (13) element of mass, stiffness, and coupling vector are given by:

$$
\begin{gathered}
m_{r l}=\int_{0}^{L}\left(\rho_{t b} A_{t b}+\rho_{p} A_{p}\right) \phi_{r}\left(x_{1}\right) \phi_{l}\left(x_{1}\right) d x_{1} \\
k_{r l}=\int_{0}^{L}\left(Y_{t b} I_{t b}+Y_{p} I_{p}\right) \phi_{r}^{\prime \prime}\left(x_{1}\right) \phi_{l}^{\prime \prime}\left(x_{1}\right) d x_{1} \\
\theta_{r}=\int_{0}^{L} J_{p} \phi_{r}^{\prime \prime}\left(x_{1}\right) d x_{1} .
\end{gathered}
$$

In the above equations, prime represents derivation with respect to $x_{1}$. Area and second area moment for the tube and piezo-plates are defined by:

$$
\begin{aligned}
\left\{A_{t b}, I_{t b}\right\} & =\iint_{t b}\left\{1, x_{3}{ }^{2}\right\} d x_{3} d x_{2} \\
\left\{A_{p}, I_{p}\right\} & =\iint_{p}\left\{1, x_{3}{ }^{2}\right\} d x_{3} d x_{2}
\end{aligned}
$$


and coupled terms defined for convenience as:

$$
J_{p+}=\iint_{p+} \frac{e_{31}}{t_{p}} x_{3} d x_{3} d x_{2}
$$

where $p+$ shows that the integral must takes place on the piezoplates in which the electric field is imposed.

In Eq. (13), electric voltage $v_{p}$ is not equal to voltage of the source $v_{x}$ because of voltage drop due to the internal resistance. To derive voltage applied on the piezo-plates we can use Eq. (10). Taking derivation with respect to time:

$$
i(t)=-\int_{A_{p}} \frac{d D_{3}}{d t} d A=\int_{A_{p}}\left(e_{31} x_{3} \frac{\partial^{3} u_{1}}{\partial t \partial x_{1}^{2}}+\epsilon_{33}^{s} \frac{\dot{v}_{p}}{t_{p}}\right) d A ;
$$

where $i(t)$ is the electric current flow in the wire connected to the electrode of the piezo-plates.

Referring to Fig. 2a and employing Kirchhoff's circuit law, the voltage on the piezo-plate's electrode is:

$$
v_{p}(t)+R i(t)=v_{s}(t) .
$$

Combining Eqs. (21) and (22), voltage of the piezo-plates is derived by the following differential equation:

$$
C_{p} \dot{v}(t)+\frac{v(t)}{R}+\{\theta\}^{t}\{\dot{a}\}=\frac{v_{s}}{R} ;
$$

where $C_{p}=\frac{\epsilon_{33}^{s} A_{p}}{t_{p}}$ is the piezo-plate capacitance. Equations (13) and (23) are coupled differential equations with the transverse displacement and voltage of the piezo-plates as unknowns.

In our case, the piezoelectric motor is driven by a sinusoidal voltage source and because Eqs. (13) and (23) are linear, we can assume:

$$
v_{s}=V_{s} e^{j \omega t}, \quad\{a\}=\{A\} e^{j \omega t}
$$

where $j$ is the imaginary unit. Substituting (24) in (13) and (23) gives:

$$
\begin{gathered}
\left(\mathbf{k}-\omega^{2} \mathbf{m}+j \omega \mathbf{d}\right)\{A\}=\{\theta\} V ; \\
R_{e q}^{-1} V=-j \omega\{\theta\}^{t}\{A\}+R^{-1} V_{s} .
\end{gathered}
$$

Substituting piezo-plate voltage from Eq. (26) at (25) gives:

$$
\{A\}=\left[\mathbf{k}-\omega^{2} \mathbf{m}+j \omega \tilde{\mathbf{d}}\right]^{-1}\{\theta\} \frac{R_{e q}}{R} V_{s} ;
$$

where $\tilde{\mathbf{d}}=\mathbf{d}+R_{e q}\{\theta\}\{\theta\}^{t}$ is the electromechanical damping and $R_{e q}=\left(\frac{1}{R}+j C_{p} \omega\right)^{-1}$.

The solution of Eq. (27) provides us the generalized coordinates which depend linearly on the amplitude of voltage source. In addition, natural frequencies of the system are the solution in which the determinant of $\mathbf{k}-\omega^{2} \mathbf{m}+j \omega \tilde{\mathbf{d}}$ becomes zero.

\section{STATOR DESIGN}

One of the main drawbacks of ultrasonic motors is their relativity low speed in comparison to electromagnetic motors. Investigating and identifying parameters that enhance the speed

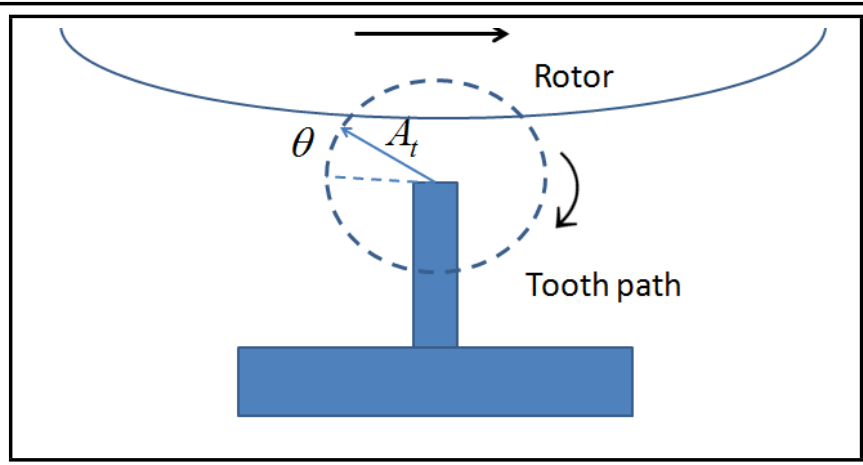

Figure 3: Tooth displacement and contact with moving rotor.

of ultrasonic motors is crucial to expand their potential applications in the various fields. The functionality and performance of the ultrasonic motors are defined and affected substantially by their structural shape and dimensions. In the following, we are interested in identifying the significant geometrical parameters' effect on the speed of the motor.

Generally, in the standing wave ultrasonic motors, the tooth of the stator follows an elliptical path as shown in Fig. 3. ${ }^{1}$ During some portion of this path, the tooth contacts with the moving rotor. Linear speed of the rotor is always lower than the maximum linear speed of the tooth. As shown in Fig. 3, the speed of the tooth is:

$$
V_{c}=A_{t} \omega_{n r} \sin (\theta)
$$

where $V_{c}$ is the velocity of the tooth displacement parallel to the rotor motion, $A_{t}$ is the displacement amplitude of the tooth, and $\omega_{n r}$ is the working frequency of the stator. When the tooth begins to contact the rotor, its speed is lower or equal to the speed of the rotor. The first case is stable while the last case is unstable. In the stable case, because the speed of the rotor is lower than the rotor speed, contact of the tooth with the rotor reduces the speed of the rotor. On the other hand, the speed of the tooth increases with time. Therefore, there is a point, namely $\theta_{e}$, in which the speeds of the rotor and the tooth become equal. As a result, the speed of the rotor will be:

$$
\omega_{r}=2 \frac{A_{t}}{a_{t b}} \omega_{n r} \sin \theta_{e} .
$$

\subsection{Objective Function}

The value of $\theta_{e}$ in Eq. (29) depends on many factors such as the material of the tooth and rotor, contact properties, and especially the preload force. ${ }^{1}$ Analytical derivation of this angle is very complicated and involves nonlinear differential equations. However, it is well known that the preload force reduces speed while increasing output torque. Therefore, by having insight about the effect of the preload, the effect of the preload is not considered as a design variable.

The remaining three terms in Eq. (29) significantly influence the speed of the motor. According to this equation, the speed of the motor is directly related to the displacement of the tooth as well as working frequency and inversely related to the width of the tube. To achieve the highest possible speed, working 
frequency of the motor should be close to the fundamental natural frequency of the stator. For any arbitrary material and dimensions of the stator, the modeling methodology outlined in section 2.2 could be utilized to calculate the natural frequency as well as the displacement of the stator on its ends. Therefore, the following function is defined as the objective function which gives maximum speed in RPM units:

$$
F=\frac{60}{\pi} \frac{A_{t}}{a_{t b}} \omega_{n r}
$$

\subsection{The Design Variables}

Although material characteristics of the tube and piezoplates influence the speed of the motor (usually due to the practical consideration and easiness of prediction of its impact on overall behavior of the motor) the, material of the motor is excluded from the design space. Brass, because of its easy machinability, considerably high density, and resistivity to oxidation is chosen as the tube material. Lead zirconate titanate, PZT is used as piezo-plate.

The structural parameters that significantly influence the velocity are chosen as length $L_{t b}$ and width $W_{t b}$ of the tube, length $L_{p}$, and width $W_{p}$ of the piezo-plates, and finally thickness $t$ of the tube as shown in Fig. 2. Because length and width of the piezo-plates must always be less than the tube length and width, instead of the piezo-plate length and width, the ratio of them to corresponding length and width of the tube are considered. In addition, because one of the main advantages of ultrasonic motors is their ability to miniaturize, the range of design variables are chosen to be in millimeter range with the lower bound imposed by a fabrication limit as listed below:
Length of tube [mm]:
$10 \leq L_{t b} \leq 30$
Width of tube [mm]:
$2 \leq W_{t b} \leq 5$
Lenght ratio $L_{p} / L_{t b}$ :
$0 \leq L_{p} / L_{t b} \leq 1$
Width ratio $4 W_{p} / W_{t b}$ :
$0 \leq W_{p} / W_{t b} \leq 1$
Thickness of tube $[\mathrm{mm}]: \quad 0.2 \leq t \leq 0.5$.

\subsection{Global Sensitivity Analysis: Morris Method}

To analyze the effect of the design variables on the objective function defined in Eq. (30) Morris method is employed. ${ }^{17}$ This method is a global sensitivity analysis method that demonstrates the overall effect of each parameter on an objective function in a given design space. This method requires computing of the elementary effects defined by:

$$
d_{i, j}(\mathbf{X})=\frac{f\left(X_{1}, X_{2}, \ldots, X_{i}+\Delta_{j}, \ldots, X_{k}\right)-f(\mathbf{X})}{\Delta_{j}} ;
$$

where $\mathbf{X}$ is a normalized vector of the variable, where the original value of parameter $x_{i}$ is given by $x_{i}=X_{i}\left(x_{i}{ }^{\max }-\right.$ $\left.x_{i}{ }^{\mathrm{min}}\right)+x_{i}{ }^{\mathrm{min}}$, and $k$ is the number of parameters. $\Delta$ is the resolution of sampling and is $\Delta_{j}=\frac{j}{m-1}$ where $j=1,2, \ldots, m$ and $m$ is the number of levels.

In Morris method for each parameter there are two factors, mean $\mu_{i}$ and variance $\sigma_{i}$, that show the overall influence of parameter and an estimate of the nonlinear and higher order effect of parameter on an objective function, respectively. These two

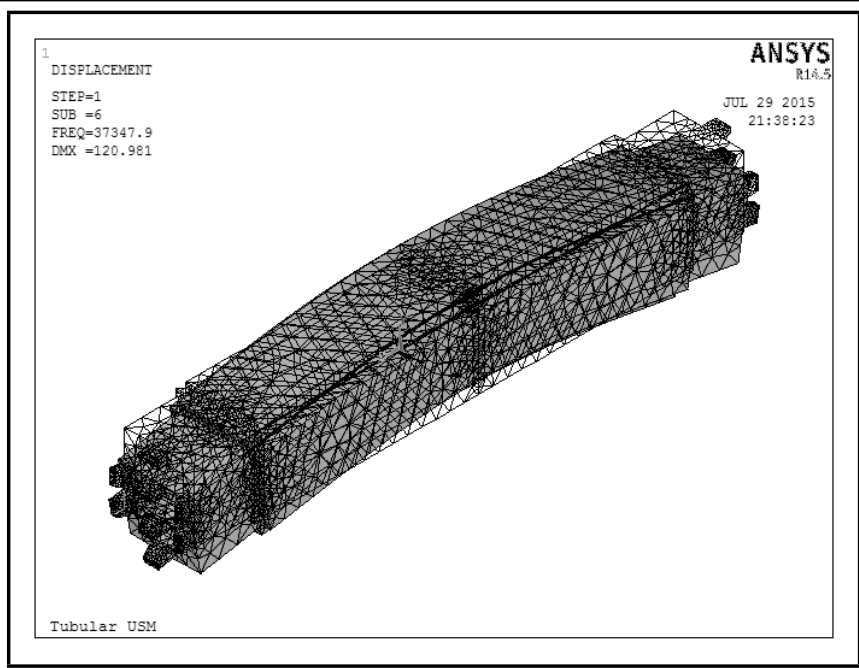

Figure 4: Working mode shape of the motor (First bending mode).

factors are defined by:

$$
\begin{gathered}
\mu_{i}=\frac{1}{m} \sum_{j=1}^{m} d_{i, j}(\mathbf{X}) ; \\
\sigma_{i}=\sqrt{m \sum_{j=1}^{m}\left(d_{i, j}(\mathbf{X})-\mu_{i}\right)^{2}} .
\end{gathered}
$$

\subsection{Modeling, Verification and Sensitivity Analysis}

The effect of the design variables on the speed of the motor is complicated and could not be intuitionally figured out. Therefore, a modeling methodology outlined in section 2 is employed. All the parameters required in the modeling are listed in Table 1. Note that the value of the damping ratio is selected tentatively as $\zeta=0.05$ and its experimental value depends on the prototype adhesive layer and the tube structural damping. For validation of the analytical model, a finite element model of the stator is constructed. Figure 4 shows the first bending mode of the stator at the frequency of $37.34 \mathrm{kHz}$ and the tooth displacement of $1.13 \mu \mathrm{m}$ obtained by FEM mode. Corresponding results by the analytical method, Eq. (27), is $37.61 \mathrm{kHz}$ and $1.13 \mu \mathrm{m}$ which is very close to the FEM model.

Using an analytical model, Eq. (28), and Morris sensitivity analysis, Eqs. (32) and, (33), the effect of the design variables on the speed, Eq. (30), is explored in Fig. 5. An important and useful result illustrated in this figure is that, surprisingly, the length of the tube does not have any significant effect on the speed of the motor. It can be justified that during the variation of the tube length, other parameters remain constant which means that the length of the piezo-plate to the length of the tube remains constant, too. Increasing the length of the stator causes the natural frequency and reduction of the motor's stiffness. Furthermore, increasing the piezo-plate length increases developed bending moment by piezoelectric which yield higher deflection. The overall effect of the length on the natural frequency and deflection counteract each other. 
Table 1: Mechanical and electrical properties of brass, PZT and voltage source, and dimensions of the motor.

Mechanical properties

\begin{tabular}{|c|c|c|c|}
\hline \multicolumn{2}{|c|}{ Brass } & \multicolumn{2}{c|}{ PZT } \\
\hline Density $\left[\mathrm{kg} / \mathrm{m}^{3}\right]$ & Modulus of elasticity [Gpa] & Density $\left[\mathrm{kg} / \mathrm{m}^{3}\right]$ & Modulus of elasticity [Gpa] \\
\hline 8450 & 97 & 7800 & 66 \\
\hline
\end{tabular}

Dimension [mm]

\begin{tabular}{||c|c|c|c|c|}
\hline \hline Length of tube & Length of PZT-plate & Width of tube & Width of PZT-plate & Thickness of tube \\
\hline 15.4 & 11.5 & 2 & 2 & 0.3 \\
\hline
\end{tabular}

Other parameters

\begin{tabular}{||c|c|c|c|c|c|}
\hline $\begin{array}{c}\text { Piezoelectric stress } \\
\text { constant } e_{31}\left[\mathrm{C} / \mathrm{m}^{2}\right]\end{array}$ & $\begin{array}{c}\text { Piezoelectric strain } \\
\text { constant } d_{31}[\mathrm{pm} / \text { Volt }]\end{array}$ & $\begin{array}{c}\text { Thickness of } \\
\mathrm{PZT}[\mathrm{mm}]\end{array}$ & $\begin{array}{c}\text { Source internal } \\
\text { resistance R[ } \Omega]\end{array}$ & $\begin{array}{c}\text { PZT capacitance } \\
\text { per unit area }\left[\mathrm{pF} / \mathrm{mm}^{2}\right]\end{array}$ & $\begin{array}{c}\text { Voltage of } \\
\text { source }[\mathrm{V}]\end{array}$ \\
\hline-12.54 & -190 & 0.267 & 50 & 60 & 10 \\
\hline
\end{tabular}

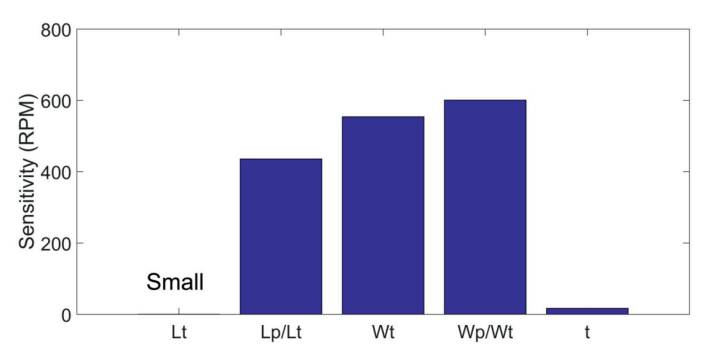

(a)

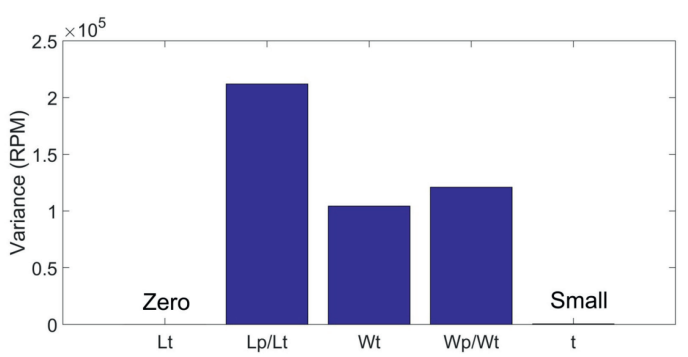

(b)

Figure 5: Morris sensitivity analysis for investigation of the stator dimension on the motor speed.

Figure $5 b$ shows variance of the elementary elements. Result of this figure shows that, except length and thickness of the tube, other parameters have considerable variance which can be the sign of nonlinearity and second order effect of these parameters on the motor speed.

Figure 6 shows variation of the speed with respected to the design variables normalized in their range. Again, there is a negligible effect of the tube length and thickness on the speed. Ratio variation of the piezo-plate to the tube length shows an optimum point at approximately $80 \%$ of the stator length. In addition, according to the result of this figure, approximately $80 \%$ of maximum speed could be achieved with a PZT length half the tube length. Effect of width ratio on the motor speed is almost linear. The speed of the motor reduces rapidly as the width of the stator increases. The width of the tube is main parameter of the tube that significantly affects the motor speed.

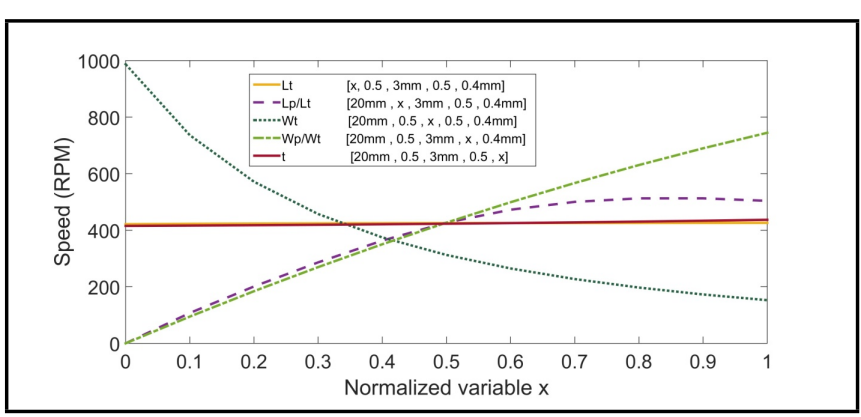

Figure 6: Variation of the motor speed with respected to the normalized design variables.

According to the result, the following remarks, which aim to enhance the speed of the motor, could be concluded:

- Length and thickness of the stator could be disregarded as design variables because of their negligible effect on the motor speed

- Width of the motor is a crucial parameter and minimum value is recommended to achieve the highest speed

- Optimum length of the piezo-plate is about $80 \%$ of the tube length

- Width of the piezo-plate must be close to tube width

In a more simplified case, length and width ratio are equal to one. In this case, speed of the motor only related inversely to the tube width. Therefore, Eq. (29) could be written in a simpler form as follows:

$$
\omega_{r}=\frac{\Omega}{a_{t b}}
$$

where $\Omega$ is the speed coefficient with unit of rad.m/s. This parameter for stators, which is constructed by the same materials and imposed with same preload force, is identical.

\section{EXPERIMENTAL RESULTS}

To validate the analytical model, a prototype of the motor with dimensions listed in Table 1 is fabricated. Precise machining and tight tolerance is controlled during fabrication. After 


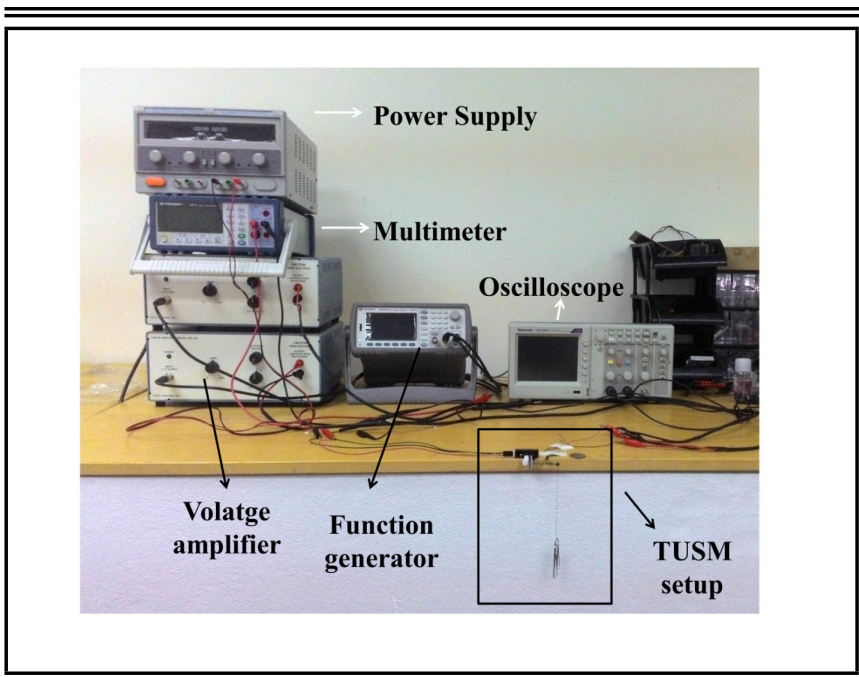

Figure 7: Equipment used for measurement and excitation of the motor.

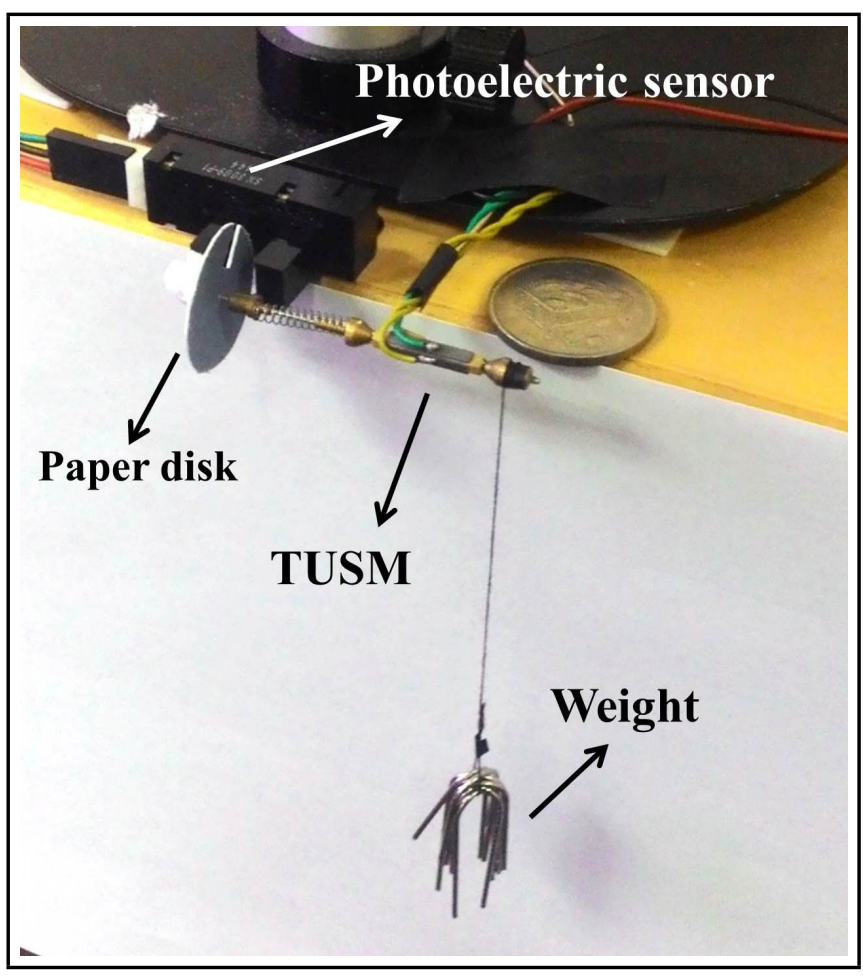

Figure 8: Prototype of the motor and experimental set-up for measuring working characteristics of the motor.

careful cutting of PZT-plates, a cyanoacrylate adhesive is utilized to bond the PZT-plates to the tube surface. Before gluing, the surface of the tube is cleaned and polished to remove oil and other contaminants and to ensure strong bonding between the PZT-plates and the tube.

The equipment employed for the measuring and exciting of the motor includes two-channel function generator Agilent 33500B, high voltage amplifier piezo-system EPA-104, oscilloscope, and multi-meter which is shown in Fig. 7. A prototype of the motor and the set-up for measuring speed and torque of the motor is shown in Fig. 8. A photoelectric sensor is used to measure speed of the motor. For measuring the torque, a fine string connected to a small weight is utilized.

For measuring natural frequency of the stator one of the

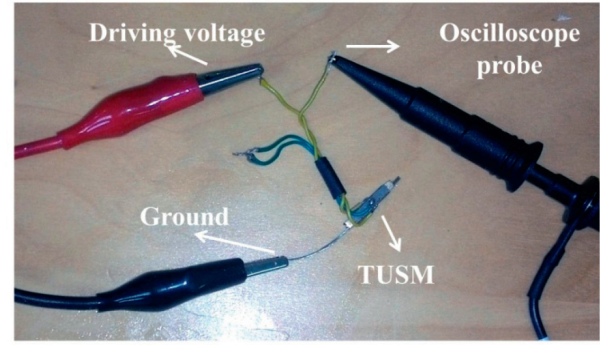

(a)

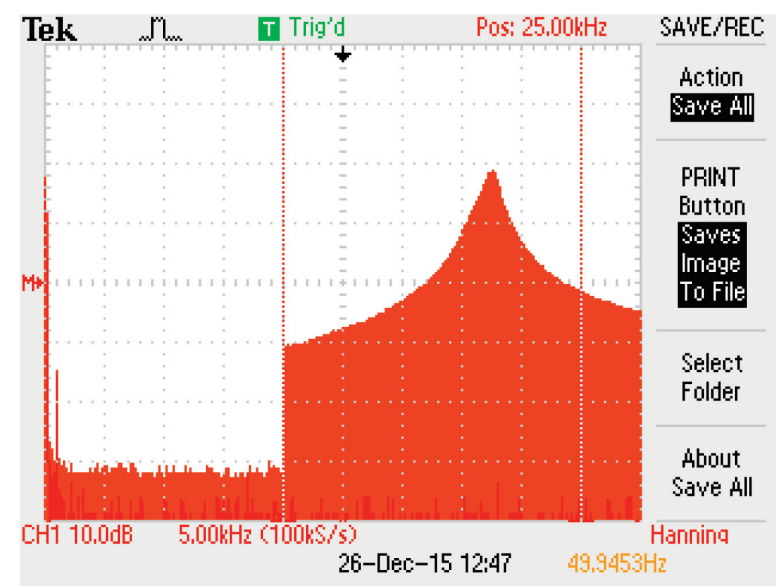

(b)

Figure 9: Set-up for measuring natural frequency of the stator, (a) one piezo-plate is connected to voltage source and another opposite plate is connected to oscilloscope probe, (b) FFT of voltage measurement of PZT-plate: vertical axis voltage in $\mathrm{dB}$ unit and horizontal axis is frequency.

piezo-plates is connected to a sinusoidal voltage and generated voltage at opposite piezo-plate is measured using an oscilloscope as shown in Fig. 9a. According to the piezoelectricity theory, this voltage is proportional to the strain imposed on the piezo-plate and, therefore, in resonance, this voltage must be maximum. In order to reduce temperature and nonlinearity effects, a sinusoidal voltage of 10 volt is applied on one PZTplate and its frequency is varied between 20 to $70 \mathrm{kHz}$. Results have been shown in Fig. 9b with peak frequency of $37.4 \mathrm{kHz}$. Compared to the theoretical result of $37.61 \mathrm{kHz}$, the result is very close. Measured voltage at the resonant is approximately 6 volt. This voltage can be related to the tooth displacement using piezoelectric constitutive equations and Euler-Bernoulli beam model as described in the appendix A. Employing this method gives $0.52 \mu \mathrm{m}$. To calculate analytical model, damping ratio of the structure is required. For measuring damping of the stator, half power method is applied. ${ }^{17}$ The natural frequency of the stator is $37.4 \mathrm{kHz}$ and the frequency where half power is observed is 35.6 and $38.2 \mathrm{kHz}$. Therefore, using $\zeta=\frac{\Delta f}{2 f_{n}}$ we have a damping ratio of 0.034 . Repeating the calculation with $\zeta=0.034$ and considering just one PZT-plate under electric field results in $0.53 \mu \mathrm{m}$ which demonstrates excellent agreement.

Stall torque of the motor is measured using the weights connected with a fine string to the end of the motor. The motor is 


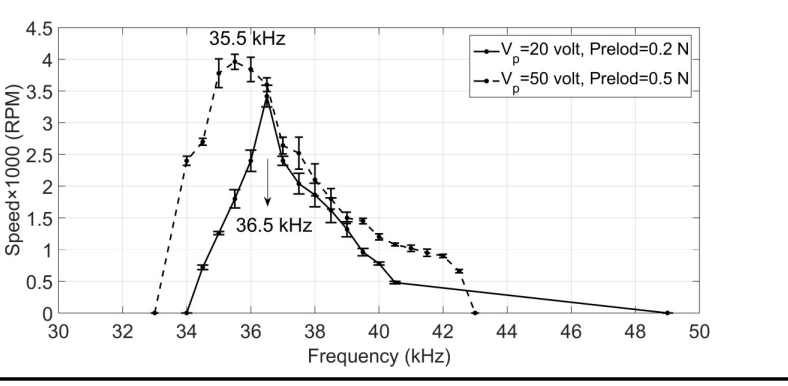

Figure 10: Motor rotational speed versus frequency, Solid line: $V_{p}=20 \mathrm{~V}$ and preload of $0.2 \mathrm{~N}$, Dash line: $V_{p}=50 \mathrm{~V}$ and preload of $0.5 \mathrm{~N}$.

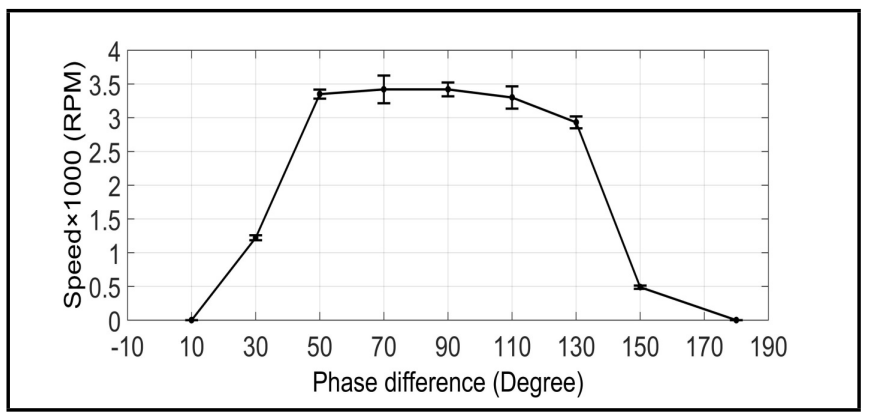

Figure 11: Motor rotational speed versus phase difference (Working frequency $36.5 \mathrm{kHz}$ ).

tested with two preloads of 0.2 and $0.5 \mathrm{~N}$ with applied voltage of 20 and $50 \mathrm{~V}$ pick voltage, respectively. Figure 10 shows the speed versus frequency of driving voltage. As shown in this figure, due to the temperate rise of the stator, working frequency is reduced from $37.4 \mathrm{kHz}$ to $36.5 \mathrm{kHz}$ for applied voltage of $20 \mathrm{~V}$ pick and $35.5 \mathrm{kHz}$ for applied voltage of $50 \mathrm{~V}$ pick. In addition, the damping ratio also rises to 0.05 . As seen in this figure, maximum rotational speed with applied voltage of 20 and $50 \mathrm{~V}$ is close to $3,500 \mathrm{rpm}$ and 4,000 rpm. Compared to the results achieved with other researchers, the designed motor achieves significantly higher speed. For instance, the motor designed by Park and $\mathrm{He}^{8}$ and Zhang et al. ${ }^{6}$ achieved up to 1,000 and 3,000 rpm with driving frequency of 50 and $55 \mathrm{~V}$, respectively. The length of the motor designed in this paper is close to that of the motor designed by Park and $\mathrm{He}$, and three times that of the motor designed by Zhang et al. It is worth mentioning that according to Eq. (30) it is expected that by miniaturization of the motor, its speed increases and the result obtained by Zhang was expectable, while, the motor designed in this paper achieved higher speed with bigger size than Zhang's motor.

Figure 11 shows the velocity versus phase difference for applied voltage of $20 \mathrm{~V}$ and preload of $0.2 \mathrm{~N}$. According to our expectation, a higher velocity is achieved when the phase difference is close to $90^{\circ}$.

Figure 12 presents motor speed versus applied voltage. As seen, start-up voltage depends on applied preload. By raising voltage, motor speed changes linearly at the beginning and almost remains constant at the end portion of the speed-voltage diagram. The reason of not rising speed is mainly because of the temperature and damping rise as well as changing of the natural frequency of the stator. ${ }^{18}$

Result of measurement reveals that for a preload of $0.2 \mathrm{~N}$

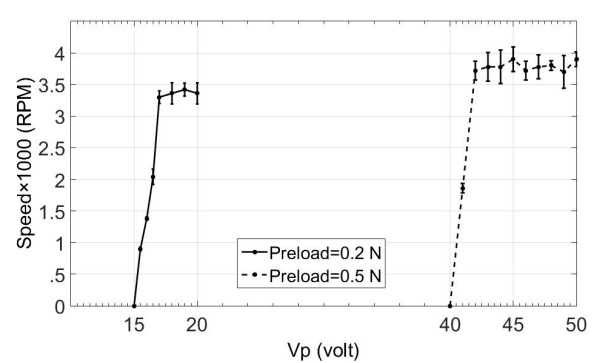

Figure 12: Motor speed versus applied voltage, Solid line: $V_{p}=20 \mathrm{~V}$ and preload of $0.2 \mathrm{~N}$ and Working frequency $=36.5 \mathrm{kHz}$, Dash line: $V_{p}=50 \mathrm{~V}$ and preload of $0.5 \mathrm{~N}$ and Working frequency $=35.5 \mathrm{kHz}$.

with a maximum applied voltage of $20 \mathrm{~V}$, current flow is $14.3 \mathrm{~mA}$ and stall torque is $54 \mu \mathrm{Nm}$ which pull 3.4 gram up. For a preload of $0.5 \mathrm{~N}$ and maximum applied voltage of $50 \mathrm{~V}$, current is $37 \mathrm{~mA}$ and stall toque is around $78.8 \mu \mathrm{Nm}$ and 4.96 gram.

\section{CONCLUSIONS}

This paper concerns electromechanical stator modeling of a tubular ultrasonic motor. The model is verified with experimental and FEM results and is used for identifying the effect of the design variables including length of the tube, length ratio of piezo-plate to the tube, width of tube, width ratio of piezoplate to the tube, and thickness of the tube on speed of the motor. Results demonstrated that speed of motor is almost independent of the tube length for a constant piezo-plate to tube length ratio and mainly determined by width of tube. This result could greatly facilitate design stage of a stator. A set of guidelines for design of a high speed ultrasonic motor is proposed and accordingly a prototype is fabricated. The motor yields very high speed which verifies the method introduced in this paper.

\section{APPENDIX A}

Piezoelectric constitutive equations for sensory application are as follows: ${ }^{19}$

$$
\begin{gathered}
S_{1}=Y_{p}^{-1} T_{1}+d_{31} \frac{v_{p}}{t_{p}} \\
D_{3}=d_{31} T_{1}+\epsilon_{33}^{s} \frac{v_{p}}{t_{p}}
\end{gathered}
$$

where $d_{31}$ is the piezoelectric strain constant. In a case that piezoelectric voltage is measured by an oscilloscope, because of high impedance of the oscilloscope input, electric displacement of the piezoelectric plate in Eq. (A.2) could be neglected. Substituting $T_{1}$ from (A.2) to Eq. (A.1) we have:

$$
\bar{S}_{1}=\frac{\varepsilon_{33}^{s}+d_{31}^{2}}{Y_{p} d_{31}} \frac{v_{m}}{t_{p}} ;
$$

where $v_{m}$ is the measured voltage. In above equation $\bar{S}_{1}$ is the average strain sensed by a piezo-plate. We can find approximated relationship between the average strain and tooth displacement using Euler-Bernoulli beam theory and assuming 
that the stator vibrating mode is close to first bending mode of free-free beam, Eq. (12) then we will have

$$
\begin{aligned}
\bar{S}_{1}= & \frac{1}{L_{p}} \int_{\left(L_{t}-L_{p}\right) / 2}^{\left(L_{t}+L_{p}\right) / 2} \frac{a_{t b}}{2} \frac{\partial^{2} u\left(x_{1}\right)}{\partial^{2} x_{1}} d x_{1}= \\
& \left.A_{e} \frac{a_{t b}}{L_{p}} \frac{\partial \phi_{1}\left(x_{1}\right)}{\partial x_{1}}\right|_{x_{1}=\left(L_{t}+L_{p}\right) / 2} ;
\end{aligned}
$$

where $A_{e}$ is an unknown. Using equation (A.3) and (A.4) and considering that the tooth displacement is $\delta=A_{e} \phi(0)$ one can find the tooth displacement by measuring voltage generated on the piezo-plate. For the case that length of the piezo-plate is equal to the tube length, tooth displacement could be calculated by:

$$
\delta=\frac{1}{4.7} \frac{L_{t b}^{2}}{a_{t b}} \frac{\varepsilon_{33}^{s}+d_{31}^{2}}{Y_{p} d_{31}} \frac{v_{m}}{t_{p}} .
$$

\section{A. ACKNOWLEDGEMENTS}

The authors would like to acknowledge the University of Malaya for providing the Research Grant (UMRG) No. RP001B-13AET.

\section{REFERENCES}

1 Sashida, T. and Kenjo, T. An introduction to ultrasonic motors. Oxford University Press, New York, NY, (1993).

2 Morita, T., Kurosawa, M. K., and Higuchi, T. A cylindrical shaped micro ultrasonic motor utilizing PZT thin film $(1.4 \mathrm{~mm}$ in diameter and $5.0 \mathrm{~mm}$ long stator transducer), Sensors and Actuators A, 83, 225-230, (2000). https://dx.doi.org/10.1016/S0924-4247(99)00388-X

3 Morita, T., Kurosawa, M. K., and Higuchi, T. An ultrasonic micromotor using a bending vibration transducer based on PZT thin film, Sensors and Actuators A, 50 (1-2), 75-80, (1995). https://dx.doi.org/10.1016/0924-4247(96)80087-2

4 Koc, B., Cagatay, S., and Uchino, K. A piezoelectric motor using two orthogonal bending modes of a hollow cylinder, IEEE Transactions on Ultrasonics, Ferroelectrics, and Frequency Control, 49 (4), 495-500, (2002). https://dx.doi.org/10.1109/58.996568

5 Kanda, T., Makino, A., Suzumori, K., Morita, T., and Kurosawa, M. K. A cylindrical micro ultrasonic motor using a micro-machined bulk piezoelectric transducer, IEEE Ultrasonics Symposium, 1298-1301, (2004). https://dx.doi.org/10.1109/ULTSYM.2004.1418028

6 Zhang, H., Dong, S.-X., Zhang, S.-Y, Wang, T.H., Zhang, Z.-N., and Fan, L. Ultrasonic micromotor using miniature piezoelectric tube with diameter of $1.0 \mathrm{~mm}$, Ultrasonics, 44, 603-606, (2006). https://dx.doi.org/10.1016/j.ultras.2006.05.064

7 He, S., Chiarot, P. R., and Park, S. A single vibration mode tubular piezoelectric ultrasonic motor, IEEE Transactions on Ultrasonics, Ferroelectrics, and Frequency Control, 58 (5), 1049-1061, (2011). https://dx.doi.org/10.1109/TUFFC.2011.1905
8 Park, S. and He, S. Standing wave brass-PZT square tubular ultrasonic motor, Ultrasonics, 52 (7), 880-889, (2012). https://dx.doi.org/10.1016/j.ultras.2012.02.010

9 Xing, Z., He, B., Xu, K., Wang, J., and Dong, S. A miniature cylindrical piezoelectric motor with an asymmetric vibrator, IEEE Transactions on Ultrasonics, Ferroelectrics, and Frequency Control, 60 (7), 1498-1504, (2013). https://dx.doi.org/10.1109/TUFFC.2013.2722

10 Pons, J. L., Rodríguez, H., Fernández, J. F., Villegas, M, and Seco, F. Parametrical optimisation of ultrasonic motors, Sensors and Actuators A, 107 (2), 169-182, (2003). https://dx.doi.org/10.1016/S0924-4247(03)00345-5

11 Zhao, C., Li, Z., and Huang, W. Optimal design of the stator of a three-DOF ultrasonic motor, Sensors and Actuators A, 121 (2), 494-499, (2005). https://dx.doi.org/10.1016/j.sna.2005.03.010

12 Ko, H.-P., Kimb, S., Kanga, C.-Y., Kima, H.-J., and Yoona, S. J. Optimization of a piezoelectric linear motor in terms of the contact parameters, Materials Chemistry and Physics, 90 (2-3), 322-326, (2005). https://dx.doi.org/10.1016/j.matchemphys.2004.09.041

13 Zhu, H., Li, Z., and Zhao, C. An efficient approach to optimize the vibration mode of bar-type ultrasonic motors, Ultrasonics, 50 (4-5), 491-495, (2010). https://dx.doi.org/10.1016/j.ultras.2009.10.010

14 Zhang, J.-T., Zhu, H., Zhou, S.-Q., and Zhao C.-S. Optimal design of a rod shape ultrasonic motor using sequential quadratic programming and finite element method, $\mathrm{Fi}$ nite Elements in Analysis and Design, 59, 11-17, (2012). https://dx.doi.org/10.1016/j.finel.2012.04.011

15 Crandall, S. H., Karnopp, D. C., Kurtz, E. F., and PridmoreBrown, D. C. Dynamics of mechanical and electromechanical systems. McGraw-Hill, New York, (1968).

16 Meirovitch, L. Fundamentals of vibrations. Waveland Pr Inc, Long Grove, IL, (2010).

17 Morris, M. D. Factorial sampling plans for preliminary computational experiments, Technometrics, 33 (2), 161174, (1991). https://dx.doi.org/10.2307/1269043

18 Xu, X., Liang, Y. C., Lee, H. P., Lin, W. Z., Lim, S. P., Lee, K. H., and Shi, X. H. Mechanical modeling of longitudinal oscillation ultrasonic motors and temperature effect analysis, Smart Materials and Structures, 12 (4), 514-523, (2003). https://dx.doi.org/10.1088/0964-1726/12/4/302

19 Ikeda, T. Fundamentals of piezoelectricity. Oxford University Press, (1990). 